How Individual Differences in Working Memory and Source Monitoring matter in Susceptibility to False Memory

\author{
LaTasha R. Holden ${ }^{1,2}$ \\ Andrew R. A. Conway ${ }^{3}$ \\ Kerri A. Goodwin ${ }^{4}$
}

${ }^{1}$ Department of Psychology, Florida State University, Tallahassee, Florida

${ }^{2}$ Florida Center for Reading Research, Tallahassee, Florida

${ }^{3}$ Department of Psychology, Claremont Graduate University, Claremont, CA

${ }^{4}$ Department of Psychology, Towson University, Towson, MD

Address Correspondence To:

LaTasha R. Holden

Florida State University, Department of Psychology

1107 West Call Street

Tallahassee, FL 32306

Phone: (850) 645-2476

Email: lholden@fsu.edu

Word Count: 11,886 


\begin{abstract}
Using the DRM word list paradigm (Roediger \& McDermott, 1995) we investigated the role of individual differences in working memory capacity (WMC) and source monitoring (SM) ability in protection from false memories (FM) in recall and recognition. Both spreading activation and monitoring are cognitive processes associated with working memory (Anderson, 1983; Cantor \& Engle, 1993), and previous research demonstrates working memory’s relation to goal maintenance (Kane \& Engle, 2003) and importance for withholding irrelevant information (Conway \& Engle, 1994). However, whether higher WMC constitutes activation or monitoring and predicts increased or decreased FM production respectively, remains inconclusive (Watson et al., 2005; Peters et al., 2007; Bixter \& Daniel, 2013). When considering SM ability, a relationship has been found between $\mathrm{WMC}$ and FM in recall, suggesting that SM mediates this relation (Unsworth \& Brewer, 2010). Other work suggests that SM and WMC interact based on the role of memory monitoring in constraining task irrelevant information (Rose, 2013; Lilienthal et al., 2015). From an activation-monitoring perspective (Gallo, 2010), we investigated individual differences in WMC and SM predicting FM in recall and recognition, testing whether the relationships are additive or interactive. Our findings support moderation, suggesting that when SM ability is too high, working memory cannot work as well to monitor and constrain activation in order to reduce FM. Only when WMC was higher and SM was lower did we show a predicted decrease in FM during recognition. This work suggests that protecting mental resources in WMC is more important for constraining FM production than SM ability and we consider the implications for real world false memories and eyewitness testimony.
\end{abstract}

Keywords: individual differences, working memory, source monitoring, false memory 


\section{How Individual Differences in Working Memory and Source Monitoring matter in Susceptibility to False Memory}

In the current study, we aim to investigate the relationships between individual differences in working memory capacity (WMC), source monitoring (SM), and false memory (FM) using the DRM word list paradigm (Deese, 1959; Roediger \& McDermott, 1995). The Deese-Roediger-McDermott (DRM) paradigm has participants view several lists of words that are semantically related (e.g., bed, rest, awake, tired, dream...) to a non-presented word (e.g., sleep). In their classic study, Roediger and McDermott (1995) found that initial false recall (falsely remembering non-presented words) enhanced the rate for later false recognition (falsely recognizing non-presented words as presented ones). Because false memories involve having memories for events that never actually occurred, taking an individual differences approach to their study allows for deeper insight into the conditions when people are more or less accurate in their recollections.

Working memory is established in the literature as an individual difference construct that is important for performing well on many higher order cognitive tasks (Engle \& Kane, 2004). Based on this, one might suspect that high working memory predicts more accurate performance on other memory tasks. However, previous studies have found no relationship between WMC and FM (Watson et al., 2005) and a negative relationship, where individuals with greater WMC were less susceptible to FM in terms of lower rates of false recall and recognition (Peters et al. 2007). Additionally, it has also been shown that higher WMC individuals have fewer FMs in recall (Watson et al., 2005) and recognition (Bixter \& Daniel, 2013) but only when warned about the DRM illusion. We argue that these mixed results have been observed because there are two 
cognitive mechanisms at play in studies of WMC and FM, and these two mechanisms produce opposite patterns of relation.

On the one hand, individual differences in WMC are linked to greater spreading activation among semantically related items (Anderson, 1983) and recall of more information in free recall and fluency tasks (Rosen \& Engle, 1997). These processes should produce a positive association between WMC and FM such that individuals with greater WMC should be more susceptible to FM. On the other hand, individual differences in WMC are linked to better goal maintenance (Kane \& Engle, 2003), conflict monitoring (Unsworth \& Spillers, 2010), and source monitoring (SM; Unsworth \& Brewer, 2010). Together, these processes should produce a negative relationship between WMC and FM, such that individuals with greater WMC should be less susceptible to FM. In order to unpack this more we will frame our work within the context of both an activation-monitoring framework (AMF; Gallo, 2010) and an individual differences perspective as it relates to WMC.

\section{The Activation-Monitoring Framework}

One theoretical account for the occurrence of false memories is the ActivationMonitoring Framework (AMF), which proclaims that activation is the process of mentally activating both presented and non-presented information, whereas, monitoring involves memory modification in order to determine the origins of activated information (Gallo, 2010). The AMF asserts that during activation all items related to and including the non-presented items (or “critical items" in the DRM paradigm) are made salient. However, during monitoring, people rely on their memories of an event in order to recall the event and/or alter their memory of the event. Therefore, activation enhances false memory creation, but effective memory monitoring reduces false memory production. 
In essence, the AMF predicts that greater activation should allow for the retrieval of more information and subsequently more false information - particularly during the DRM illusion which finds the probability of false recognition (.86) to be approximately double the rate of false recall (.40) (Roediger \& McDermott, 1995). This suggests that in addition to initial encoding, both recall and recognition provide additional chances for activation, allowing for a more robust false memory effect during recognition.

In addition, the AMF posits that better monitoring should allow for the ability to search memorial information in a more accurate way—predicting that better monitoring should allow for fewer false memories. This presents an interesting puzzle for the study of false memory production from the perspective of WMC. Higher WMC could produce better monitoring of source information and in turn fewer false memories, or in contrast, due to greater capacity, higher WMC could produce greater activation, which would predict more false memories. To further discuss these contrasting predictions, we will now consider WMC and its relation to activation and monitoring.

\section{Individual Differences in Working Memory Capacity}

Baddeley and Hitch (1974) proposed a model of memory, which involved the central executive, the articulatory (phonological) loop and the visuo-spatial sketchpad. In this model, the three systems work (hence, working memory) together in order to effectively store and process information (also see Baddeley, 2000). Thus, working memory capacity (WMC) is a measure of the ability to successfully process multiple pieces of information simultaneously and WMC uses a portion of the short-term memory system involved in executive functions, control of attention, and the active maintenance of material (Miyake \& Shah, 1999). Complex WMC tasks involve the assessment of concurrent processing, updating, manipulating, and storage of information, 
typically using the operation or reading span tasks (i.e., OSPAN and RSPAN, see Turner \& Engle, 1989; Daneman \& Carpenter, 1980). Throughout the WMC literature, higher WMC span individuals are found to use more efficient forms of information processing. In fact, previous research demonstrates that the major distinction among WMC span differences is based on access to and use of cognitive resources. Higher WMC span is found to correspond with more cognitive resources, a greater capacity for focusing attention (Engle, 2002; Kane \& Engle, 2003), and better processing and performance on complex WMC span tasks which provide an assessment of one's WMC (see Unsworth et al. 2005). Performance on these complex WMC span tasks has been shown to relate to and be important for performance on a variety of longterm memory tasks and cognitive ability measures (Engle \& Kane, 2004; Delaney, Godbole, Holden \& Chang, 2017; also see Holden, Goodwin \& Conway, 2020).

Furthermore, previous research demonstrates that limitations in WMC are task independent such that greater WMC is associated with larger activation limits- that is, more cognitive resources available to use for activation in long-term memory (Cantor \& Engle, 1993). In a seminal paper, Cantor and Engle (1993) used the fan paradigm to test the idea that activation can be explained by capacity. The fan paradigm requires learning target sentences that take the form: "The subject is in the place (e.g., The lawyer is in the boat)." Each subject was given one, three, or four places of association; thus, fan size was one, three, or four. Results indicated that the time required to verify sentences was slower for three places than one and four places was slower than three. The interpretation was that if the fan is four you can allocate only $25 \%$ of overall capacity to each place node, so activation is spreading from the subject to each place. The idea is that more places require more activation being spread to each place and thus more 
capacity is required to verify the subjects with the correct place forms. This suggests that greater capacity provides more space for activation of semantic associates.

Additionally, individual differences in WMC have been shown to relate to source monitoring ability, (Ruffman et al., 2001; Unsworth \& Brewer, 2010) strategic encoding, and contextual retrieval processes (Unsworth \& Spillers, 2010). Source monitoring is the process whereby one decides about the origin of details concerning memory, knowledge, or beliefs (for a review refer to Johnson, Hashtroudi \& Lindsay, 1993). In the working memory literature, better source monitoring, more strategic encoding (i.e., learning information in a way that is most effective, enabling more accurate and easier retrieval) and greater context retrieval (i.e., relying on contextual details surrounding memory retrieval, typically enabling memory improvement) are generally linked with higher WMC span, as these are associated with more accurate performance on memory tasks (Ruffman et al., 2001; Unsworth \& Brewer, 2010; Unsworth \& Spillers, 2010).

In essence, higher span is thought to correlate with greater activation and more effective monitoring allowing for increased accuracy. In terms of FM production this would imply that even though higher WMC might be associated with greater resources allocated during activation, it does not necessarily make the subsequent memory monitoring less effective. Instead, the findings above suggest that the efficiency of memory monitoring may depend on one's allocation and capacity limitations of WM.

This idea was tested in several studies when Lilienthal et al. (2015) had participants make lexical decisions to determine whether information was relevant or irrelevant when performing a WM task. In one study, the task included an implicit measure of the activation of both currently and formerly relevant information. In another study, a recognition task was used to measure item 
and source recognition ability. They found that those with low WMC spans were not holding irrelevant information in a more active state in memory (i.e., greater activation) than high spans. Instead, low spans were simply worse at identifying information as irrelevant upon retrieval and these differences were driven by poor source monitoring. This suggests that because low spans are worse at identifying the source of information than high spans this is more of a distinguishing factor for them than differences in the activation of irrelevant information. However, the items in the lists used by Lilienthal et al. were not semantically related and thus would not necessarily predict the same outcome as typically observed in the DRM false memory research, which utilizes semantically-related word lists. This may indicate that activation is less engaged for their task than what would happen in the DRM, especially in terms of how a high or low WMC span would handle the task based on activation and monitoring. If these processes may be differently engaged in the DRM compared to the task in Lilienthal et al., then that is a key difference for thinking about whether activation is less important across high and low spans, in general.

However, we aim to investigate the relation between WM, SM, and FM through the DRM so the items and the tasks to be used in the current work are qualitatively different than those in the Lilienthal work. Moreover, additional work finds that the ability to use source information to recall relevant information and withhold irrelevant information is caused by individual differences in WM abilities and these differences also covary with other cognitive abilities, like secondary memory and fluid intelligence (Rose, 2013). Together, these studies underscore the importance of monitoring process in WM for constraining irrelevant information and suggest that WM and source monitoring abilities are likely to be important for helping constrain the production of FM. 
As we know that both spreading activation and monitoring are cognitive processes associated with working memory (Anderson, 1983; Cantor \& Engle, 1993), and previous research demonstrates working memory's relation to goal maintenance (Kane \& Engle, 2003), however, whether higher WMC predicts greater activation or decreased false memory production through better memory monitoring remains to be seen. Consequently, we focus next on WM studies exploring the importance of activation and monitoring and how these might be considered in the context of FM.

\section{WMC, Source Monitoring, and False Memory: Greater Activation or Better Monitoring?}

Prior research on the relationship between WMC and false memory has revealed mixed results. There have been reports of no relationship between WMC (on the OSPAN) and false memory using the DRM task (Watson et al., 2005). In contrast, a significant negative relationship has been reported between WMC (on the backward digit span) and false memory in both recall and recognition tasks (Peters et al. 2007). These conflicting results could indicate that the relationship between WMC and false memory is sensitive to particular task demands. In addition, there may be a discrepancy in results due to the differing assessments of WMC: OSPAN is a complex WMC span task which focuses on manipulation and retrieval, whereas, backward digit span is a simple WMC span task that focuses more on transformation and retrieval. Although these findings highlight the importance of considering differential effects in terms of FM production, task demands in WMC shall remain outside the empirical pursuit of the present work, thus we will focus our attention on research pertinent to the current work.

In their study, Unsworth and Brewer (2010) proposed that a relationship exists between WMC and FM when accounting for source-monitoring (SM) ability. Through a structural equation model derived from a battery of cognitive assessments for measuring WMC and FM, 
their results suggest that SM (measured based on the discrimination of female and male voices as the source of information) was the link between WMC and FM. Though their task battery approach for assessing relationships among constructs was advantageous, and provided the first evidence for a mechanistic link between WMC and FM, in the domain of FM the next step is to consider SM within the context of the FM task rather than having it be task independent of FM. In other words, Unsworth and Brewer assessed WMC, SM, and FM with independent tasks for each construct, but from a real-world perspective it is more valid to assess SM and FM together. For example, mechanistically, to output information from memory may require the retrieval of both information and its source simultaneously. As such, memorial features (such as source information) may be bound at the time of encoding (see Raaijmakers \& Shiffrin, 1981; Gillund \& Shiffrin, 1984). In this case, it makes sense to think of these items as related in the process of discriminating between accurate and inaccurate information. Such a process may entail sorting through memorial information and monitoring the information for inaccuracy. Taking this into consideration, we will now more carefully discuss the importance of memory monitoring in the context of FM from the AMF view.

With regard to monitoring, Gallo (2010) distinguished between diagnostic monitoring, where people rely on their expectations to make a memory decision, and disqualifying monitoring, where people rely on collateral information to make a memory decision. As Gallo deems diagnostic monitoring to be primary (i.e., less mentally taxing) and disqualifying monitoring to be secondary (i.e., more mentally taxing), one might infer that individuals with higher WM span can more easily enable both diagnostic and disqualifying monitoring and those with lower WM span may rely on diagnostic monitoring or disqualifying monitoring when making a memory decision. Furthermore, diagnostic monitoring involves using the quality and 
evidence of a memory to make a memory decision. For example, within the context of the DRM paradigm a subject may think "I remember the word bed on the list because I remember picturing dreaming while in a bed when I saw the word bed."), whereas, disqualifying monitoring is based on inconsistencies in recollections (e.g., "I don't remember seeing the word sleep because I remember trying to form a mental picture of each word on the list. I remember making a mental picture for the word dream but I never pictured the word sleep"). As such, one might presume that due to differences in attention and cognitive ability, those with higher WM span should be more thorough when making memorial decisions. To this end, the AMF would advocate that higher WMC should predict greater accuracy on monitoring tasks-resulting in fewer false memories.

Taken together, the perspective of the AMF combined with the research discussed above we arrive at a few key considerations. First, although Unsworth and Brewer (2010) proposed a compelling avenue for future research, their measure of SM shared no overlap with their FM measure. Based on the formation of real-world false memories, one should consider that source information and memorial details are entangled and thus, an alternative approach would be to embed source information within the DRM paradigm. Second, following aforementioned theories of memory binding, if the memorial and source information are bound upon encoding, then at retrieval it will be necessary to assess the memorial information in this way. As the DRM paradigm provides an ecologically valid method for assessing false memories in the research laboratory, it is a useful tool for mimicking how such processes might occur in the real world (e.g., eyewitness memory). Moreover, the majority of the aforementioned studies only considered either false recall or false recognition, we will consider relationships of WMC, SM 
and FM for both recall and recognition tasks. Hence, we arrive at the ambitions of the present work.

\section{The Current Study}

Prior work suggests that SM plays a role in activation and monitoring processes in WM (Lilienthal et al., 2015) as well as in the DRM paradigm (Unsworth \& Brewer 2010; see also Gallo 2010) but it is less clear how SM and WMC work together to affect false recall and recognition in the DRM paradigm. Unsworth and Brewer tested SM and FM with SM as a trait or general ability measure, and demonstrated that trait or general SM ability explained the role that WM plays in decreasing false recall. Lilienthal et al. examined monitoring in terms of its role within a WM task and found that individuals with higher WMC are better equipped to avoid memory errors. Lilienthal et al. explained that high WM spans are better able to accurately identify source information during retrieval and use this as a monitoring process which helps constrain the production of inaccurate or irrelevant information. In contrast, individuals with low WMC are more susceptible to memory failures because they are more likely to confuse source information during retrieval which leads to monitoring failures. Lilienthal and colleagues suggested that differences in abilities to monitor information during retrieval and qualify it as relevant or irrelevant drives differences in memory performance. Whereas Unsworth and Brewer's results suggest the role of SM as the mechanism or mediator, the results of Lilienthal et al. suggest that SM ability might interact with WM in the production of false memories.

To our knowledge, no work has assessed the roles of SM and WM together in tests of false memory with SM embedded within the DRM. In order to investigate theories of WMC and SM together in false memory, we embedded SM in the DRM by presenting DRM lists with either an audio or a visual presentation, and assessed trait WMC separately with complex span 
tasks. Based on the AMF we expected that high WMC functions in both monitoring and activation in recall and recognition. However, it is unknown according to AMF whether SM functions in both activation and monitoring in recall and recognition. The purpose of the current work is to clarify how WMC and SM affect FM and to clarify when and how they may help constrain FMs. Specifically, we sought to investigate the relationship between WMC and FM while testing SM as a potential mediator or moderator of this relation.

The goals of our study are threefold: (1) to directly re-consider the role of SM in mediating the relationship of WMC and FM, while using a more ecologically valid assessment of SM, (2) to indirectly assess the paradox of whether WMC predicts more versus less FM production based on capacity viewed as better monitoring or greater activation, and (3) to consider these relationships for both false recall and recognition using mediation and moderation models. In our study, subjects completed complex span tasks (OSPAN and RSPAN) as measures of WMC, and remembered DRM word lists for later recall and recognition. Source monitoring ability was assessed by incorporating audio and visual presentations of the DRM word lists.

When considering an activation account of false memories, higher WMC would predict less accurate recall, worse SM, and in turn, more false memories. Based on a spreading activation account we would expect that more correct recall during the DRM should predict more spreading activation making for a potential increase in FM. Based on this account, we would expect higher correct recall to be associated with more false recall, more correct recognition, and with more false recognition.

When considering a monitoring account of false memories and because we know WMC has been shown to be related to SM ability (Unsworth \& Brewer, 2010), we hypothesized that higher WMC would predict more accurate recall, better SM, and this would in turn predict fewer 
false memories. If monitoring constrains activation we expect increases in correct recall to be associated with increased SM accuracy, fewer SM errors, and fewer false recall and fewer [false] recall of other intrusions (i.e., false recall of words semantically related to the critical lure).

If spreading activation increases errors in the DRM, then an increase in activation should also cause a disruption in monitoring. Working memory entails both activation and monitoring. If working memory is activation then during the DRM task we should see higher WMC associated with higher correct recall and recognition, better SM, but also more false recall and recognition. However, WM as monitoring says that we should see fewer source monitoring errors (SME), and less false recall of critical lures and other intrusions, as well as less false recognition.

\section{Method}

\section{Participants}

A total of 127 undergraduate students were recruited from the Research Pool at a public state university. All participants were at least 18 years of age. After completing informed consent, participants were told that the study would test their memory for audio and visual information in two parts. Participants were tested in groups of up to six people for each part of the study. WMC was assessed in Part 1 and FM production was tested in Part 2 only. Participants returned for Part 2 of the study between 1 and 14 days $(M=2.8, S D=2.3)$ after Part 1 . After completing the experiment participants were debriefed and received credit toward a course requirement.

\section{Tasks}

Working Memory Capacity. To assess trait WMC participants completed the automated OSPAN and RSPAN tasks (see Unsworth et al., 2005). OSPAN requires completing a 
series of arithmetic problems while remembering a list of letters (Turner \& Engle, 1989). For example, an individual trial in a list would take the following form: $[5 \times 9]+5=45 ?$ Yes or No? $P$. Participants were instructed to solve the equation, answer yes or no, and then remember the letter for later recall. The RPSAN replaces math problems with making veridical judgments for sentences while remembering lists of letters (Daneman \& Carpenter, 1980). For example, making a veridical judgment for the sentence: "When at last his eyes opened, there was no gleam of triumph, no shade of anger" Yes or No? R. Participants were instructed to read the sentence, answer yes or no, and then remember the letter for later recall. Participants received three to seven letters per trial and three sets of each trial length, totaling 15 trials-yielding a maximum score of 75, with higher scores indicating higher WMC. The total score was calculated using the partial unit scoring method (see Conway et al., 2005).

DRM Word Lists. A modified version of the DRM task was administered. The critical modification from traditional DRM presentations was presenting the lists by either audio clip (male voice) or visually from a timed PowerPoint slideshow. During the DRM task we presented seven 15-item word lists (see Stadler, Roediger \& McDermott, 1999). The first list was presented visually as a practice list (i.e., king). The remaining six lists (i.e., chair, needle, sleep, sweet, soft, rough) were presented through either audio or visual modality. Each list contained words (e.g., bed, rest, awake, tired, dream...) semantically related to a critical, non-presented word (e.g., sleep).

Recognition and Source Monitoring Test. The recognition test contained 186 words: 90 presented words from both audio and visual lists (i.e., each of the 15 items presented in the six lists during recall), six critical lures (the semantically related lure words that were not included in list presentations from the six lists presented during recall), as well as 90 non-presented 
distractors from six other DRM word lists (i.e., music, anger, cold, bread, foot, mountain, see Stadler, Roediger \& McDermott, 1999) that were not included during initial presentation and not semantically related to list items. False recognition was the mean proportion of false alarms, identified by indicating that the "non-presented" item was seen before. Source monitoring was quantified as the mean proportion of items correctly recognized and matched with its correct source presentation (audio or visual).

\section{Design and Procedure}

We employed a within-subjects design. After completing an informed consent form, participants completed the OSPAN and the RSPAN. The span tasks were counterbalanced to control for order effects. The WMC tasks served as Part 1 of the study, and participants returned for Part 2 (see Participants section for information about length between parts 1 and 2) to receive full credit for their participation.

During Part 2 of the study, participants were given instructions on how to complete the DRM task. Unlike previous studies on WMC and FM, participants were not informed or warned about the DRM or its effect on memory (cf., Watson et al., 2005). Instead, participants were told that the task would assess their memory for audio and visual information. Participants were informed that they would complete word recall for each list and then a final recognition test of all lists learned. At the end of each word list, participants had 1.5 min to recall as many of the words from the list as possible. The order of word list presentations was counterbalanced using a Latin Square, ensuring that each source (audio and visual) occurred for each list in each possible order. We assessed correct and false recall of critical items. False recall was quantified by the percent of critical items falsely recalled across all six lists. After all lists were presented participants completed a final recognition test. 
The final recognition test was used to quantify recognition accuracy and source monitoring ability. During the recognition test, participants indicated if the item was 'Old' (i.e., previously presented) or 'New' (not previously presented), and if they selected 'Old,' participants discriminated whether the item was presented in audio or visual form. In addition, certainty ratings based on the participant's confidence in their responses were recorded on a scale from 1 (very unsure) to 5 (very sure). There was no time limit for completing the recognition test. Dependent measures on the recognition test included: the percentage of correctly recognized items (out of 90, correctly identifying presented words as presented) and the percentage of critical lures (out of 6). Source monitoring ability was measured by the percentage of correct source attributions, which is the proportion of 'Old' items with a correctly identified sourcehigher percentages of source attributions signified better source monitoring ability. After completion of both portions of the experiment (WMC portion and FM portion) participants were thanked and debriefed about the purpose of the study.

\section{Results}

\section{Analysis Plan}

Based on previous research we tested two accounts of the expected relations between WMC, SM and FM. First, and we expected that higher WMC would be associated with better SM ability which would in turn predict fewer false memories (cf. Unsworth \& Brewer, 2010). On the other hand, we expected that the role SM plays in the relation of WMC and FM is interactive in that higher spans are better able to accurately identity source information during retrieval whereas lower spans make more source errors which leads to more monitoring failures (cf. Lilienthal et al., 2015). These findings should predict fewer false memories for high spans 
relative to lower spans but Lilienthal et al.'s previous findings suggest the relation of WMC and FM may also depend on SM ability and SM errors.

We tested our hypotheses through descriptive statistics, correlations, and regression models with the mean proportions of false recall and false recognition rates as the primary outcome variables for false memory production. Regression analyses were run to investigate the role of SM in mediating or moderating the relationship of WMC and FM. The predictors were RPSAN $^{1}$, SM ability, and SM errors, and the outcome measures were false recall (FRL), and correct (CRG) and false (FRG) recognition.

\section{Descriptive Statistics and Correlations}

\section{DRM Effects}

Twelve participants were removed based on having missing working memory span scores on the WM measures (five total) or based on being identified as univariate outliers (seven total) with scores falling outside of the range of 3 SD for any of the WM, SM, and FM measures. Table 1 provides summary statistics of these data. We found the classic DRM effect of false memory with similar rates between proportions of hits and false alarms (see Roediger \& McDermott, $1995)$ in terms of correct $(M=.57, S D=.10)$. and false recall $(M=.55, S D=.27)$ and correct $(M$ $=.81, S D=.11)$ and false recognition $(M=.87, S D=.19)$. The proportion of correct source monitoring ability during recognition $(M=.71, S D=.15)$ was lower than the proportions of correct recognition and false recognition. Although the average number of source errors was moderate $(M=9.37, S D=9.17)$ the range of average source errors was high $(\operatorname{Min}=0, \operatorname{Max}=$ 50) with slightly more source errors from list items presented in the audio modality $(M=4.91$, $S D=6.64)$ compared to the visual modality $(M=4.45, S D=5.42)$ but these were not

\footnotetext{
${ }^{1}$ Based on the dissociation of the OSPAN measure in patterns of correlation (see Table 1) only RSPAN was included as a WMC measure predicting SM and FM production.
} 
significantly different $(p>.05)$. However, we found that false alarms with no source errors from list items presented in the audio modality $(M=2.27, S D=.92)$ were marginally significantly higher $(p=.069)$ than those presented in the visual modality $(M=2.06, S D=.93)$, replicating previous findings on the effect of modality on false memory (see Gallo et al., 2001; Smith et al., 2008; Smith \& Engle, 2011). Also, false alarms with source errors were higher from list items presented in the audio modality $(M=.45, S D=.79)$ than the visual modality $(M=.44, S D=.72)$ but these were not significantly different $(p>.05)$.

\section{Relations during Recall and Recognition}

Next, Table 2 reports all bivariate correlations of these data. Turning to correct recall, we found significant relations with correct recognition $r(113)=.49, p<.01$, and source monitoring ability during recognition, $r(113)=.63, p<.01$. However, we found a positive relation with false recognition for audio sources with no source errors $r(113)=.40, p<.01$ and a negative relation with false recognition for audio sources with source errors $r(113)=-.41, p<.01$, suggesting that although there was no relation between correct recall and false recognition rates overall $r(113)=$ $.03, p>.05$, when we examine false recognition based on source errors a pattern emerges indicating the importance of considering source modality in false memory ${ }^{2}$. In terms of false recall we found significant relations with overall false recognition $r(113)=.35, p<.01$, as well as false recognition with correct source for audio $r(113)=.23, p<.05$ and visual modalities, $r(113)=.25, p<.01$

\section{Working Memory}

\footnotetext{
${ }^{2}$ We also found a negative relation with correct recall and the number of source errors during recognition, $r(113)=-$ $.40, p<.01$.
} 
In terms of working memory, we found that RSPAN was a better predictor of most variables than OSPAN. Thus, we focus on RSPAN as the measure of interest for WMC in subsequent analyses. Looking at the correlations, RSPAN was positively related to correct recall $r(113)=.31, p<.01$, trending toward significantly negatively related to other recall intrusions $r(113)=-.18, p<.10$, indicating that higher WMC was associated with more correct recall and tended to be associated with fewer intrusions other than critical lures during recall. RSPAN was not related to false recall or false recognition, indicating that contrary to our predictions, higher WMC was not associated with fewer false memories during recall or recognition. Interestingly, higher WMC was also not associated with more items correctly recognized. However, RSPAN was trending toward significantly positively related to source monitoring ability during recognition, $r(113)=.17, p<.10$ and was significantly negatively related to source errors during recognition $r(113)=-.19, p<.05$. These results indicate that higher WMC was associated with better correct source identification and fewer source errors during recognition. Based on these findings, we further explored through regression models, the role of SM in the relation of WM and FM and whether the relation suggests mediation or moderation.

\section{Working Memory, Source Monitoring and False Memory Mediation Models}

False Recall

In order to assess whether SM mediates the relation between WMC and FM we ran several regression analyses. First, we ran regressions for the direct path between the predictor and outcome, followed by the indirect path of both predictors simultaneously predicting the different FM outcome variables during recall and recognition. All coefficients in the models were unstandardized. We report full model statistics for these mediation models predicting false recall and recognition in Table 3. The direct path from RSPAN to FRL and the direct path from 
SM ability to FRL were not significant. The direct path from RSPAN to SM ability was trending toward significant, indicating that higher WMC was associated with an increase in source monitoring during recognition. In the model with RSPAN and SM predicting FRL we found that neither RSPAN, nor SM ability significantly predicted false recall and the model was also not significant. These results did not provide evidence for SM ability mediating the relation between WM and false memory during recall (noting here that the FM measure was at recall and the SM measure was at recognition), thus next we tested WMC, SM ability and FM during recognition.

\section{False Recognition}

The direct path from RSPAN to FRG was not significant. The direct path from SM ability to FRG was trending toward significant, indicating that better source monitoring during recognition was associated with an increase in false recognition. The direct path from RSPAN to SM ability was also trending toward significance, indicating that higher WMC was associated with more accurate source monitoring during recognition. In the model with RSPAN, SM, and FRG we found that both RSPAN and SM ability were trending toward significance and the model was also trending toward significance (see Table 3). These model results indicate that higher WMC was associated with a marginal decrease in false recognition while greater SM ability was associated with a marginal increase in false memory during recognition.

The direct paths between WMC and FM were negative for both false recall and false recognition, indicating in both cases that higher WMC was associated with a lower probability of "retrieving" false information, but both were non-significant. In addition, the remaining paths with RSPAN and SM to the FRL and FRG models were either trending toward significance or non-significant, which together, did not provide strong support for SM mediating the relationship between WMC and FM. Overall, because the effects appeared to be very small we did not run 
additional analyses to test for mediation because of the potential lack of statistical power to detect the effects.

\section{Working Memory, Source Monitoring and False Memory Moderation Models}

In order to assess whether the relation between WMC and FM is moderated by SM, we ran several regression analyses. First, we ran regression models with the main effects of WM and SM predicting FM in recall and recognition, followed by models with main effects and the interaction of WMC and SM. We compared the main effects models to the models with main effects and interactions for significant increases in model variance. All coefficients in these models are unstandardized. We report a results summary in the text below and refer the reader to Table 4 for full model statistics for each of these analyses.

\section{False Recall}

In the main effects model of WMC and SM predicting false recall we found that neither RSPAN nor SM ability significantly predicted false recall (see Table 4). In the main effects and interaction of WMC and SM model predicting false recall, neither RSPAN, SM ability, nor their interaction significantly predicted false recall (see Table 4). The model was also nonsignificant. When we compared the main effects only to the main effects and interaction model we found no significant difference in variance accounted for between these models.

\section{False Recognition}

In the main effects model of WMC and SM predicting false recognition we found that both RSPAN and SM ability trending toward significantly predicting false recognition — with RSPAN negatively predicting and SM ability positively predicting. The model was also trending toward significance (see Table 4). The main effects and interaction model of WM and SM predicting false recognition was significant, with SM ability trending toward significant, and 
with WM and the interaction statistically significant (see Table 4). When we compared the main effects only to the main effects and interaction model we found a significant increase in variance accounted for between these models. This suggests that the main effects and interaction model is a better fitting model compared to the main effects only model. This also provides evidence in support for the idea that SM ability moderates the relation of WM and FM in recognition, noting that only when WMC was higher and SM ability was lower did we see a predicted decrease in FM production during recognition (see Figure 1). Based on these findings we further unpacked the interaction. Using the Johnson-Neyman method for simple slope analysis (see Figure 2), results indicated that only when WMC on the RSPAN was average, $b=0.23, t(111)=1.91, p=$ .0590 or at least $+1 S D$ above average, $b=0.48, t(111)=3.02, p<.01$ did we find trending or significant slopes for the relation of SM ability and false recognition. When RSPAN was at least -1 SD below average the slope of SM ability predicting false recognition was not significant, $b=$ $-0.03, t(111)=-0.17, p>.05$. These results indicate that when WMC is average or above there is an increasingly positive relation between SM ability and false recognition, that is, better SM ability predicts more false recognition when WM is higher.

\section{Discussion}

The purpose of this study was to examine the relationship of WMC and FM while also exploring SM as a potential mediator or moderator of this relation. We replicated the DRM effect with comparable rates of false recall and recognition as previous work (Roediger \& McDermott, 1995). We hypothesized that false memory in recall and recognition would be related to WMC and SM ability. Specifically, WMC and FM were expected to be negatively related, and although we did observe negative trends between RSPAN and false recall, other intrusions in recall, and false recognition, all of these relations were small or statistically nonsignificant. 
It was also expected that WMC and SM ability would be positively related and that WMC and SM errors would be negatively related. As predicted, there was a positive but nonsignificant trend between RSPAN and SM ability, in that higher RSPAN scores predicted better SM ability. We also found a negative relation between WMC (via RSPAN) and SM errors, indicating that higher RSPAN scores helped participants to reduce errors in SM during recognition. OSPAN was not significantly related to any of the SM or FM measures which is in line with previous work using OSPAN as a WMC measure when exploring FM (Watson et al. 2005; also see Peters et al. 2007).

We provided support for the hypotheses that there are significant relationships among WMC, SM, and FMs, when WM is assessed via the RPSAN. Our findings suggest that WM was most predictive of avoiding other intrusions in recall but the DRM illusion appeared robust which may have offset potential benefits of WMC for constraining the production of false memories in either recall or recognition. Instead, we found that WMC was more predictive and seemingly more important for the production of correct information in recall and recognition and in supporting veridical details like identifying source information and constraining source errors in recognition.

We expected SM ability and FM to be negatively related and SM ability and correct memories to be positively related. We found no relationship between SM ability and FM in recall or recognition. However, we did find strong positive relations between SM ability and correct memories in both correct recall and recognition, suggesting that the SM ability mechanism may work harder in order to support the production of and identification of correct memories as opposed to constraining the production of false memories. We also expected SM errors and FM to be positively related and SM errors and correct memories to be negatively related. We found 
no significant relation between SM errors and false memories in false recall or recognition. We did find however, that SM errors and correct memories were negatively related in correct recall but interestingly, they were not related to correct recognition. This provides additional support for the idea that the monitoring mechanism works more to help produce correct information in recall than to identify it through recognition or to constrain production of false information.

Moreover, the patterns of relation revealed in the current work also stress the distinction between production versus identification in retrieval. That is, production (e.g., free recall) is typically thought to be harder than identification (e.g., cued recall or recognition) but there are additional concerns about suggestibility and familiarity involved in recognition during the DRM from the vantage of WMC and SM abilities. For example, the additional resources that higher WMC provides alongside additional opportunities for activation based on having a higher SM ability could be too much to control in the context of highly semantically related information like that presented in the DRM. Perhaps together these two abilities in the context of highly semantically related information causes too many opportunities for increased activation and this overwhelms the potential benefits of the monitoring mechanisms involved in higher WMC and SM abilities. Additionally, the patterns of relation that we saw in Figure 1 revealed that even with lower WMC and higher SM ability this did not reduce FM production as much as high WMC and lower SM ability, suggesting again the importance of freeing up mental space so that WMC can work to constrain activation.

Based on previous findings in the literature, WMC seems to play an important role in helping people distinguish relevant information from irrelevant information (Lilienthal et al., 2015; Rose, 2013). Specifically, Lilienthal et al. suggested that more source errors should happen in lower WMC span individuals because of the inability to identify relevant from irrelevant 
information. Therefore, it is reasonable to expect that if a negative relation was found between WMC and source errors then this might come about based on monitoring failures. In contrast, if a positive relation was found between $\mathrm{WMC}$ and source errors, activation could be playing more of a role such that higher span individuals have more space to activate more information. Considering this idea in light of our finding of a stronger negative relation between WMC and source errors and a weaker positive relation between WMC and source monitoring ability, our results suggest that the current work replicates Lilienthal et al.'s work. That is, in the context of the DRM higher WMC span is more important for monitoring and constraining source errors possibly through more accurately separating relevant from irrelevant information. Our work suggests that in the context of the DRM, WMC is more predictive and thus potentially more important for monitoring and reducing source errors than for spreading activation in order to retrieve more source information.

Considering the summary of relations with SM ability and SM errors, our results suggest that when the monitoring mechanism breaks down and source errors occur they are produced from monitoring failures. Moreover, these monitoring failures appear to be in opposition to producing correct information (we also note that SM ability and SM errors were weakly negatively related). Because SM errors and correct recognition were not related we surmised this was the case because SM errors were more related to WMC and SM ability. Thus, it may be the that higher WMC and SM abilities are more important for producing accurate memories. Further, when the monitoring components of these processes fail or are limited, this indicates a breakdown in the ability to produce the correct information but may not necessarily help much to constrain processes like false recall and false recognition. 
Observing false recall and recognition rates similar to those in previous studies suggests that although the DRM illusion is robust, there are still potential benefits of WMC and SM for constraining inaccuracies or monitoring failures which lead to the production of false memories. We believe this may be the case based on the strength of the patterns of correlations of the WM and SM measures and the correct recall and correct recognition measures but additional experimental work is required in order to determine causal mechanisms.

In this study, we also expected that SM ability mediates and/or moderates the relation of WM and FM. However, unlike previous work (Unsworth \& Brewer, 2010) we did not find evidence for the notion that SM mediates the relation of WMC and FM in either false recall or recognition. Because we did not find evidence of strong relations between WMC and FM in recall or recognition there was not much of an effect to mediate. Instead, we found evidence that the relation between WMC and FM is moderated by SM ability, but only in false recognition. We observed significant positive relations between SM ability and FM when WMC was average to high but we did not a find significant relation when WM was below average. Moreover, although the WMC and SM measures appeared to be most important for helping to produce correct or veridical information and details, the finding of moderation also suggests that there are certain conditions when these abilities help to constrain the production of false information. As aforementioned, both WMC and SM abilities help us arrive at correct memories but our results suggest that when both are too high there are higher rates of false memory production in recognition compared to if people were only high in ability on either WMC or SM. Additionally, we found that false memories during recognition were the lowest when WMC was high and SM ability was below average, suggesting that potential monitoring benefits of higher WMC may function optimally for constraining false memory under these conditions. That is, under 
conditions of high WMC and SM abilities being better able to correctly identify the source information associated with the context of the memory could spread activation more. So in turn, this could predict more rather than less FMs. Our data cannot speak further to this but specifically asking higher spans to use their WMC to monitor more closely might help uncover whether this is the case when WM and SM are considered during the DRM because without the warning (see also Watson et al., 2005), having greater span may not necessarily be a good thing for limiting false memories (our data suggests this specifically for limiting false recognition).

Because our results provide support for opposing dual-path mechanisms for exploring the relations between WMC, SM, and FM, we feel that the AMF provides the best explanation for the results (Gallo, 2010) although it is not fully explanatory. The AMF considers memory performance based on both a generative and conservative perspective - activation as any process associated with mentally activating non-presented items and in turn, contributing to the production of false information and monitoring as any process associated with memorial editing and decisions, thereby contributing to the reduction of false information. For example, the AMF posits that monitoring involves purposeful and controlled processing of information in order to achieve accuracy. Working memory literature suggests individuals with high spans are recognized for using more controlled and purposeful processing of information, perhaps resulting in more effective memory monitoring (Engle, 2002; Kane \& Engle, 2003). To this end, the AMF would advocate that individuals with higher WMC should perform source discrimination tasks embedded within the DRM paradigm with a greater degree of accuracy relative to those with lower WMC. Although we did not find a significant negative relation of RSPAN and false recognition, our results support the hypothesis of greater WMC as better monitoring because we did find a positive trend between RSPAN and SM ability and a significant negative relation 
between RSPAN and SM errors. Thus, because those with higher span have more cognitive resources at their disposal, theoretically, they could employ either diagnostic, disqualifying monitoring or both as needed to balance accurate source and memory retrieval while avoiding monitoring errors.

The results of the present study also demonstrate that a higher WMC advantage may have also led participants to become overtaxed during the retrieval process. In this case, individuals with higher spans may have eventually exhausted their cognitive resources during portions of the recognition test and subsequently, had to rely on more diagnostic monitoring to make their memory decisions. This could also explain why higher RSPAN was related to more accurate recall, and had a positive trend with SM but was not significantly related to lower rates of false recall or recognition. The recognition test forced participants to make 'Old' or 'New' judgments in addition to discriminating source information and making confidence ratings for each item. Perhaps the combination of these three tasks and the length of the recognition test exhausted the cognitive resources that usually serve as an advantage to those with higher span scores.

In line with the view of higher WMC meaning greater activation, it might be that higher WMC span leads participants to think more about what was encoded, thereby activating more false information relative to low spans. Previous work demonstrates this type of pattern in the need for cognition paradigms (see Graham, 2007) in which people who enjoy thinking more are also more susceptible to false memories. However, it is important to note that the AMF does not articulate thorough predictions to sort out different ways that spreading activation might happen during encoding, recall, source monitoring/identification and recognition.

Regarding the DRM illusion and the notion that higher capacity equates to greater activation, the results of the present study would also support the theory of "more is less" (Toglia 
et al., 1999). In their work, Toglia et al. found that as more information was recalled overall accuracy was less, due to the higher likelihood of false memory recall. In our work this indicates that successful monitoring in addition to the advantage of higher WMC was not enough to help participants completely avoid the effect of the DRM illusion in recall or recognition. Because the recognition test forces participants to discriminate among several items, whereas, recall only necessitates the production of information, the recognition test could have required the participants to rely more heavily on monitoring processes than during recall. Moreover, in the context of AMF, our findings suggest that recognition may require the use of more disqualifying monitoring. As we know, disqualifying monitoring is "secondary" (Gallo et al., 2010, p. 837), meaning it is more mentally taxing, it may be the case that higher WMC allows for greater activation and better monitoring, but together these may overstretch the limits of the memory system. In our study, perhaps higher WMC allowed for greater activation, then necessitating the use for stronger monitoring during recognition overtaxed the limitations of the memorial system, in essence, demonstrating a "more is less" phenomenon.

\section{Limitations}

The present study is not without limitations. First, one might argue that embedding the SM task within the DRM paradigm makes it difficult to isolate SM ability as a baseline measure from correct recognition. However, the purpose for embedding the SM task within the DRM was to mimic the occurrence of these processes outside the research laboratory —in the real world it would be difficult to isolate a memory from its source information. Previous research also supports the notion of binding the memorial and feature information in this way (cf., Raaijmakers \& Shiffrin, 1981; Gillund \& Shiffrin, 1984). Because there is an abundance of previous research demonstrating that the memorial system functions in this way, our approach is 
valid both in and outside the research laboratory. In addition, previous research that assessed modality effects within the DRM did not separate the source test from the recognition of items (e.g., Pierce et al., 2005). Second, in spite of the fact that the recognition tests were counterbalanced, perhaps the length and complexity of the tests promoted increased levels of fatigue which neither higher nor lower span individuals could avoid, taxing valuable cognitive resources in WMC that could aid monitoring and decrease FM production. Also, the present study did not further investigate the differences in recognition or SM ability based on information presented in the audio and visual modalities. There is evidence for reducing false memories for high spans when information is presented visually rather than in an auditory fashion, (Smith et al., 2008; Smith \& Engle, 2011) however, these studies did not assess individual differences in a trait or general SM ability. Future research should further investigate the modality effect with regard to WM span, trait SM ability and false memory production.

In our design, we failed to show the same pattern of results for the OSPAN and RSPAN measures. While considering previous findings with OSPAN as pertains to DRM effects (e.g., Watson et al., 2005), it is important to note that we observed a dissociation in the behavior of two WMC measures that are considered to be very widely used and, presumably behaviorally compatible. To our knowledge, only a few studies have observed such a dissociation in the behavior of these two measures and specifically with the RSPAN measure demonstrating effects where the OSPAN does not. These studies suspect the dissociation to pertain to aspects of the processing components involved in the tasks (Chow et al., 2016; Macnamara et al., 2011). However, there is some evidence in previous research to suggest that the low between subject variability in certain experimental or behavioral tasks might contribute to the low reliability of individual differences and correlational approaches (Hedge et al., 2018). We mention this 
because the current work employs a classic behavioral paradigm which tends to have low between subject variability which could have also undermined our individual differences approach in exploring the relations of WM and SM in FM production.

\section{Conclusion}

In sum, the results of the present study suggest that there is a slight advantage for individuals with higher WMC in combating the DRM illusion; however, this advantage is moderated by SM ability and was not strong enough to eliminate the effects of the illusion (Watson et al., 2005). Likewise, the results of the present study did not provide support for the notion that SM ability plays a mediating role in reducing susceptibility to false memories (cf. Unsworth \& Brewer, 2010), specifically for those higher in WM span. The results of the present study also imply that there are dual paths involved mechanistically in the production of false memories. That is, according to the WMC as monitoring view higher span is associated with a slight advantage in combating false memory production; whereas, the WMC as activation view finds the higher span advantage associated with greater false memory production. Our results also demonstrate both the duality of WM span and the robustness of the false memory illusion even when considering individual differences in SM ability. In spite of the variety of cognitive advantages that higher WM and SM abilities bring, including the more accurate memories, these results also suggest that high accuracy is not always associated with fewer false memories. Specifically, our results suggest that there may be certain conditions which help constrain FM more than others when WM and SM are considered together.

It appears that both WMC and SM ability play important roles in investigating false memories. Mechanistically, we have demonstrated that the cognitive process that facilitates false memory production is very complex, underlining the complexity of memory in general. In the 
current work our overarching question was whether having a more accurate and effective memory system would predict fewer false memories depending on whether memory capacity was viewed as activation or as monitoring. The present results emphasize the importance of designing contexts wisely to help reduce FM production by constraining familiarity and reducing contextual details that promote suggestibility and spread activation as well as finding ways to free up mental space as much as possible in order to let working memory work. We showed that correct recall does predict monitoring abilities and failures in recall. This suggests that the hard part of memory production is predictable from monitoring but the correct recognition and identification part of memory is not as predictable from a monitoring perspective. These results urge considering how to make contexts best for helping everyone be more apt to reduce false memory production. This is key in light of eyewitness testimony being so heavily relied upon outside the research laboratory. Moreover, creating the contexts to help reduce FM becomes even more important considering the roles of person perception and culpability in the context of eyewitness testimony. As such, we should create contexts to help people constrain processes involved in suggestibility, and familiarity or gist-based forms of retrieval.

In the present paper, we have provided evidence supporting the claim that memorial virtues like higher WMC span may facilitate more accurate recall and help constrain errors in source discrimination. However, this very virtue may also facilitate more false recall and recognition of information. For this reason, we feel that future work should continue to investigate the relationship between WMC and false memory production based on dual mechanisms. Taken together, we can conclude that memory's vices are indeed virtues enabling us to process, store, manipulate and retrieve information (see Schacter, 2001) and moreover, the 
fallibility of the memorial system emerges when we believe that its utility always necessitates precision.

\section{Acknowledgments}

The authors are grateful to Gaby Escalante, Wendy Zhao, and Kaitlin Ensor for assistance with data collection and processing.

\section{Funding}

The first author is supported by funding through the Provost's Postdoctoral Fellowship Program at Florida State University.

\section{Disclosure Statement}

The authors declared no potential conflicts of interest with respect to the research, authorship, and/or publication of this article.

\section{Data Availability Statement}

The datasets generated and/or analyzed during the current study are available from the corresponding author on reasonable request. 


\section{References}

Anderson, J. R. (1983). A spreading activation theory of memory. Journal of Verbal Learning and Verbal Behavior, 22, 261-295.

Baddeley, A. D., \& Hitch, G. J. (1974). Working memory. In G. A. Bower (Ed.), The psychology of learning and motivation: Advances in research and theory (Vol. 8, pp. 47-90). New York: Academic Press.

Baddeley, A. D. (2000). "The episodic buffer: a new component of working memory?". Trends in Cognitive Science, 4, 417-423.

Bixter, M. T., \& Daniel, F. (2013). Working memory differences in illusory recollection of critical lures. Memory \& cognition, 41, 716-725.

Cantor, J., \& Engle, R.W. (1993). Working memory capacity as long-term memory activation: An individual differences approach. Journal of Experimental Psychology: Learning, Memory, and Cognition, 5, 1101-1114.

Chow, M.A., Macnamara, B., \& Conway, A.R.A. (2016). Phonological similarity in working memory span tasks. Memory \& Cognition, 44, 937-949.

Conway, R. A., \& Engle, R. W. (1994). Working memory and retrieval: a resource-dependent inhibition model. Journal of Experimental Psychology: General, 4, 354-373.

Conway, A. R., Kane, M. J., Bunting, M. F., Hambrick, D. Z., Wilhelm, O., \&amp; Engle, R. W. (2005). Working memory span tasks: A methodological review and user's guide. Psychonomic Bulletin \&amp; Review, 12, 769-786.

Daneman, M. \& Carpenter, P. A. (1980). Individual differences in working memory and reading. Journal of Verbal Learning and Verbal Behavior, 19, 450-466. 
Deese, J. (1959). On the prediction of occurrence of particular verbal intrusions in immediate recall. Journal of Experimental Psychology, 58, 17-22.

Delaney, P. F., Godbole, N. R., Holden, L. R., \& Chang, Y. (2017). Working memory capacity and the spacing effect in cued recall. Memory, 26, 784-797.

Engle, R. W. (2002) Working memory capacity as executive attention. Current Directions in Psychological Science, 11, 19-24.

Engle, R. W., \& Kane, M. J. (2004). Executive attention, working memory capacity, and a twofactor theory of cognitive control. Psychology of learning and motivation, 44, 145-200.

Gallo, D. A., McDermott, K. B., Percer, J. M., \& Roediger, H. L., III. (2001). Modality effects in false recall and false recognition. Journal of Experimental Psychology: Learning, Memory, and Cognition, 27, 339-353.

Gallo, D. A. (2010). False memories and fantastic beliefs: 15 years of the DRM illusion. Memory \& Cognition, 38, 833-848.

Gillund, G. \& Shiffrin, R.M. (1984). A retrieval model for both recognition and recall. Psychological Review, 91, 1-67.

Graham, L. M. (2007). Need for cognition and false memory in the Deese-RoedigerMcDermott paradigm. Personality \& Individual Differences, 42, 409-418.

Hedge, C., Powell, G., \& Sumner P. (2018). The reliability paradox: Why robust cognitive tasks do not produce reliable individual differences. Behavioral Research Methods, 50, 1166-1186.

Holden, L. R., Goodwin, K. A., \& Conway, A.R.A. (2020, September). Converging Evidence that Higher Trait Working Memory Capacity aids Standardized Test Performance under 
Race Related Stereotype Threat. PsyArXiv Preprint. Retrieved from: https://psyarxiv.com/ckuwx.

Johnson, M. K., Hashtroudi, S., \& Lindsay, D. S. (1993). Source monitoring. Psychological Bulletin, 114, 3-28.

Kane, M. J., \& Engle, R. W. (2003). Working-memory capacity and control of attention: The contributions of goal neglect, response competition, and task set to Stroop interference. Journal of Experimental Psychology: General, 132, 47-70.

Lilienthal, L., Rose, N. S., Tamez, E., Myerson, J., \& Hale, S. (2015). Individuals with low working memory spans show greater interference from irrelevant information because of poor source monitoring, not greater activation. Memory \& Cognition, 43, 357-366.

Macnamara, B. N., Moore, A. B., \& Conway, A. R. A. (2011). Phonological similarity effects in simple and complex span tasks. Memory \& Cognition, 39, 1174-1186.

Miyake, A., \& Shah, P. (1999). Models of working memory: Mechanisms of active maintenance and executive control. New York: University Press.

Peters, M. J. V., Jelicic, M., Verbeek, H., \& Merckelbach, H. (2007) Poor working memory predicts false memories, European Journal of Cognitive Psychology, $19,213-232$.

Pierce, B. H., Gallo, D. A., Weiss, J. A., \& Schacter, D. L. (2005). The modality effect in false recognition: Evidence for test-based monitoring. Memory \& Cognition, 33, 1407-1413.

Raaijmakers, J. G. W., \& Shiffrin, R. M. (1981). Search of associative memory. Psychological Review, 88, 93-134. 
Roediger, H. L. III, \& McDermott, K. B. (1995). Creating false memories: Remembering words not presented in lists. Journal of Experimental Psychology: Learning, Memory, and Cognition, 21, 803-814.

Rose, N. S. (2013). Individual differences in working memory, secondary memory, and fluid intelligence: Evidence from the levels-of-processing span task. Canadian Journal of Experimental Psychology, 67, 260.

Rosen, V.M., \& Engle, R.W. (1997). The role of working memory capacity in retrieval. Journal of Experimental Psychology: General, 126, 211-227.

Ruffman, T., Rustin, C., Garnham, W., \& Parkin, A. J. (2001). Source monitoring and false memories in children: Relation to certainty and executive functioning. Journal of Experimental Child Psychology, 80, 95-111.

Schacter, D. (2001). The seven sins of memory: How the mind forgets and remembers. Boston, MA, US: Houghton, Mifflin and Company.

Smith, R. E., \& Engle, R. W. (2011). Study modality and false recall: The influence of resource availability. Experimental Psychology, 58, 117-124.

Smith, R. E., Hunt, R. R., \& Gallagher, M. P. (2008). The effect of study modality on false recognition. Memory \& Cognition, 36, 1439-1449.

Stadler, M. A., Roediger, H. L., \& McDermott, K. B. (1999). Norms for word lists that create false memories. Memory \& Cognition, 27, 494-500.

Toglia, M. P., Neuschatz, J. S., \& Goodwin, K. A. (1999). Recall accuracy and illusory memories: When more is less. Memory, 7, 233-256.

Turner, M. L., \& Engle, R. W. (1989). Is working memory capacity task dependent? Journal of Memory and Language, 28, 127-154. 
Unsworth, N., \& Brewer, G. A. (2010). Individual differences in false recall: A latent variable analysis. Journal of Memory and Language, 62, 19-34.

Unsworth, N., \& Spillers, G. J. (2010). Variation in working memory capacity and episodic recall: The contributions of strategic encoding and contextual retrieval. Psychonomic Bulletin \& Review, 17, 200-205.

Unsworth, N., Heitz, R. P., Schrock, J. C., \& Engle, R. W. (2005). An automated version of the operation span task. Behavior Research Methods, 37, 498-505.

Watson, J. M., Poole, B. J., Bunting, M. F., \& Conway, A. R. A. (2005). Individual differences in susceptibility to false memory in the Deese-Roediger-McDermott paradigm. Journal of Experimental Psychology: Learning, Memory, and Cognition, 31, 76-85. 
Table 1. Descriptive statistics of all measures.

\begin{tabular}{lrrrr}
\hline Measure & \multicolumn{1}{c}{ M } & \multicolumn{1}{c}{ Min } & Max \\
OSPAN & & & & \\
RSPAN & 56.26 & 13.89 & 10.0 & 75.0 \\
CRL & 51.57 & 12.49 & 14.0 & 74.0 \\
FRL & .57 & .10 & .27 & .77 \\
OtherFRL & .55 & .27 & 0.0 & 1.0 \\
CRG & 1.79 & 1.9 & 0.0 & 9.0 \\
FRG & .81 & .11 & .49 & .99 \\
SM & .87 & .19 & .33 & 1.0 \\
SME & .71 & .15 & .27 & .94 \\
VASE & 9.37 & 9.17 & 0.0 & 50.0 \\
AVSE & 4.45 & 5.42 & 0.0 & 28.0 \\
FAAA & 4.91 & 6.64 & 0.0 & 34.0 \\
FAVV & 2.27 & .92 & 0.0 & 3.0 \\
FAAV & 2.06 & .93 & 0.0 & 3.0 \\
FAVA & .45 & .79 & 0.0 & 3.0 \\
MC & .44 & .72 & 0.0 & 2.0 \\
& 4.42 & .50 & 2.68 & 5.0 \\
\hline
\end{tabular}

Note. $\mathrm{N}=115$; OSPAN $=$ operation span; RSPAN = reading span;

$\mathrm{CRL}=$ proportion correct recall; $\mathrm{FRL}=$ proportion false recall; OtherFRL $=$ other intrusions false recall; $\mathrm{CRG}=$ proportion correct recognition; $\mathrm{FRG}=$ proportion false recognition;

$\mathrm{SM}=$ proportion correct source monitoring ability; $\mathrm{SME}=$ source monitoring errors total; VASE = visual reported audio source errors; AVSE = audio reported visual source errors; FAAA $=$ false alarm recognition audio source correct; FAVV = false alarm recognition visual source correct; FAAV = false alarm recognition audio reported visual source error; $\mathrm{FAVA}=$ false alarm recognition visual reported audio source error; $\mathrm{MC}=$ mean confidence 
Table 2. Correlations for all measures.

\begin{tabular}{|c|c|c|c|c|c|c|c|c|c|c|c|c|c|c|c|}
\hline Measure & 1 & 2 & 3 & 4 & 5 & 6 & 7 & 8 & 9 & 10 & 11 & 12 & 13 & 14 & 15 \\
\hline 1. OSPAN & -- & & & & & & & & & & & & & & \\
\hline \multirow[t]{2}{*}{ 2. RSPAN } & $.54 * *$ & -- & & & & & & & & & & & & & \\
\hline & {$[.39, .66]$} & & & & & & & & & & & & & & \\
\hline \multirow[t]{2}{*}{ 3. CRL } & 0.09 & $.31 * *$ & -- & & & & & & & & & & & & \\
\hline & {$[-.10, .27]$} & {$[.14, .47]$} & & & & & & & & & & & & & \\
\hline \multirow[t]{2}{*}{ 4. FRL } & -0.02 & -0.09 & 0.15 & -- & & & & & & & & & & & \\
\hline & {$[-.20, .17]$} & {$[-.27, .09]$} & {$[-.04, .32]$} & & & & & & & & & & & & \\
\hline \multirow[t]{2}{*}{ 5. OtherFRL } & -0.11 & $-0.18 \dagger$ & $-.20^{*}$ & -0.01 & -- & & & & & & & & & & \\
\hline & {$[-.29, .07]$} & {$[-.35, .01]$} & {$[-.37,-.02]$} & {$[-.19, .18]$} & & & & & & & & & & & \\
\hline \multirow[t]{2}{*}{ 6. CRG } & 0.13 & 0.05 & $.49^{* *}$ & 0.05 & 0.01 & -- & & & & & & & & & \\
\hline & {$[-.05, .31]$} & {$[-.13, .23]$} & {$[.33, .61]$} & {$[-.13, .24]$} & {$[-.18, .19]$} & & & & & & & & & & \\
\hline \multirow[t]{2}{*}{ 7. FRG } & -0.03 & -0.13 & 0.03 & $.35^{* *}$ & -0.03 & $.32 * *$ & -- & & & & & & & & \\
\hline & {$[-.21, .15]$} & {$[-.30, .06]$} & {$[-.15, .21]$} & {$[.18, .50]$} & {$[-.21, .15]$} & {$[.14, .47]$} & & & & & & & & & \\
\hline \multirow[t]{2}{*}{ 8. SM } & 0.06 & $0.17 \dagger$ & $.63^{* *}$ & 0.03 & 0.0016 & $.72 * *$ & 0.15 & -- & & & & & & & \\
\hline & {$[-.12, .25]$} & {$[-.01, .34]$} & {$[.51, .73]$} & {$[-.15, .21]$} & {$[-.18, .18]$} & {$[.61, .80]$} & {$[-.03, .33]$} & & & & & & & & \\
\hline \multirow[t]{2}{*}{ 9. SME } & 0.04 & $-.19^{*}$ & $-.40^{* *}$ & 0.01 & 0.0029 & 0.02 & 0.12 & $-.68^{* *}$ & -- & & & & & & \\
\hline & {$[-.14, .23]$} & {$[-.36,-.01]$} & {$[-.54,-.23]$} & {$[-.17, .20]$} & {$[-.18, .19]$} & {$[-.16, .20]$} & {$[-.07, .29]$} & {$[-.77,-.57]$} & & & & & & & \\
\hline \multirow[t]{2}{*}{ 10. VASE } & 0.12 & -0.09 & $-0.18 \dagger$ & 0.1 & -0.03 & 0.06 & 0.1 & $-.44 * *$ & $.70^{* *}$ & -- & & & & & \\
\hline & {$[-.07, .29]$} & {$[-.27, .10]$} & {$[-.35, .00]$} & {$[-.08, .28]$} & {$[-.21, .15]$} & {$[-.12, .24]$} & {$[-.09, .27]$} & {$[-.58,-.28]$} & {$[.59, .78]$} & & & & & & \\
\hline \multirow[t]{2}{*}{ 11. AVSE } & -0.03 & $-.19^{*}$ & $-.40^{* *}$ & -0.06 & 0.03 & -0.02 & 0.08 & $-.58 * *$ & $.81^{* *}$ & 0.15 & -- & & & & \\
\hline & {$[-.22, .15]$} & {$[-.36,-.01]$} & {$[-.54,-.23]$} & {$[-.24, .12]$} & {$[-.15, .21]$} & {$[-.20, .16]$} & {$[-.10, .26]$} & {$[-.69,-.45]$} & {$[.74, .87]$} & {$[-.04, .32]$} & & & & & \\
\hline \multirow[t]{2}{*}{ 12. FAAA } & -0.01 & 0.02 & $.40^{* *}$ & $.23 *$ & 0.02 & $.32 * *$ & $.40 * *$ & $.55^{* *}$ & $-.45 * *$ & -0.07 & $-.56^{* *}$ & -- & & & \\
\hline & {$[-.19, .17]$} & {$[-.16, .20]$} & {$[.24, .55]$} & {$[.05, .39]$} & {$[-.17, .20]$} & {$[.15, .48]$} & {$[.24, .55]$} & {$[.41, .67]$} & {$[-.58,-.29]$} & {$[-.25, .11]$} & {$[-.67,-.42]$} & & & & \\
\hline \multirow[t]{2}{*}{ 13. FAVV } & -0.05 & -0.02 & 0.03 & $.25 * *$ & -0.06 & 0.08 & $.58 * *$ & $.19^{*}$ & $-.19 *$ & $-.37 * *$ & 0.04 & 0.13 & -- & & \\
\hline & {$[-.23, .14]$} & {$[-.20, .16]$} & {$[-.16, .21]$} & {$[.07, .41]$} & {$[-.24, .13]$} & {$[-.11, .26]$} & {$[.44, .69]$} & {$[.01, .36]$} & {$[-.36,-.00]$} & {$[-.51,-.20]$} & {$[-.14, .22]$} & {$[-.05, .31]$} & & & \\
\hline \multirow[t]{2}{*}{ 14. FAAV } & -0.03 & -0.13 & $-.41 * *$ & -0.10 & -0.02 & -0.10 & 0.06 & $-.45 * *$ & $.54^{* *}$ & 0.06 & $.70^{* *}$ & $-.80^{* *}$ & -0.002 & -- & \\
\hline & {$[-.21, .15]$} & {$[-.31, .05]$} & {$[-.55,-.25]$} & {$[--28, .08]$} & {$[-.21, .16]$} & {$[-.27, .09]$} & {$[-.12, .24]$} & {$[-.59,-.29]$} & {$[.40, .66]$} & {$[-.13, .24]$} & {$[.60, .78]$} & {$[-.86,-.72]$} & {$[-.19, .18]$} & & \\
\hline \multirow[t]{2}{*}{ 15. FAVA } & 0.06 & -0.05 & -0.05 & 0.05 & 0.03 & 0.09 & $.24 * *$ & $-.22 *$ & $.40^{* *}$ & $.65^{* *}$ & 0.02 & 0.06 & $-.56^{* *}$ & 0.03 & -- \\
\hline & {$[-.12, .24]$} & {$[-.23, .13]$} & {$[-.23, .14]$} & {$[-.13, .23]$} & {$[-.15, .21]$} & {$[-.10, .27]$} & {$[.06, .41]$} & {$[-.39,-.04]$} & {$[.24, .54]$} & {$[.53, .75]$} & {$[-.16, .20]$} & {$[-.13, .24]$} & {$[-.67,-.41]$} & {$[-.15, .21]$} & \\
\hline \multirow[t]{2}{*}{ 16. MC } & 0.01 & -0.03 & $0.17 \dagger$ & $.25^{* *}$ & 0.03 & -0.08 & $-0.16 \dagger$ & $.22 *$ & $-.40^{* *}$ & $-0.16 \dagger$ & $-.42 * *$ & $.19^{*}$ & 0.02 & $-.36 * *$ & -0.13 \\
\hline & {$[-.18, .19]$} & {$[-.21, .15]$} & {$[-.02, .34]$} & {$[.07, .42]$} & {$[-.15, .21]$} & {$[-.26, .11]$} & {$[-.33, .02]$} & {$[.04, .38]$} & {$[-.54,-.23]$} & {$[-.33, .02]$} & {$[-.56,-.25]$} & {$[.01, .36]$} & {$[-.16, .20]$} & {$[-.51,-.19]$} & {$[-.31, .05]$} \\
\hline
\end{tabular}

Note. $\mathrm{N}=115$; OSPAN $=$ operation span; RSPAN = reading span;

$\mathrm{CRL}=$ proportion correct recall; FRL = proportion false recall; OtherFRL = other intrusions false recall; $\mathrm{CRG}$ $=$ proportion correct recognition; $F R G=$ proportion false recognition;

$\mathrm{SM}=$ proportion correct source monitoring ability; SME = source monitoring errors total;

VASE = visual reported audio source errors; AVSE = audio reported visual source errors;

FAAA = false alarm recognition audio source correct; FAVV = false alarm recognition visual source correct;

FAAV $=$ false alarm recognition audio reported visual source error;

$\mathrm{FAVA}=$ false alarm recognition visual reported audio source error; $\mathrm{MC}=$ mean confidence $\dagger p<.10, * p<.05, * * p<.01$, Values in square brackets indicate the $95 \%$ confidence interval for each correlation. 
Table 3. WMC and Source Monitoring Ability Mediation Models for False Recall and False Recognition

Mediation Multiple Regression Summary Table

\begin{tabular}{|c|c|c|c|c|c|c|c|c|c|c|}
\hline \multirow[b]{3}{*}{ Predictor(s) } & \multicolumn{5}{|c|}{ Mediator } & \multicolumn{5}{|c|}{ Dependent Measure } \\
\hline & \multicolumn{5}{|c|}{ Source Monitoring (SM) } & \multicolumn{5}{|c|}{ False Recall } \\
\hline & $b(S E)$ & $t(d f)$ & $p$ & $F(d f)$ & Multiple $r 2$ & $b(S E)$ & $t(d f)$ & $p$ & $F(d f)$ & Multiple $r 2$ \\
\hline \multicolumn{11}{|l|}{ Direct Effects } \\
\hline RSPAN & $0.0020(.0011)$ & $1.85(113)$ & $=0.066$ & $(1,113)=3.44$ & 0.030 & $-0.0020(.0020)$ & $-0.99(113)$ & $>.05$ & $(1,113)=.097$ & 0.0084 \\
\hline SM & -- & -- & -- & -- & -- & $0.054(.17)$ & $0.31(113)$ & $>.05$ & $(1,113)=.96$ & 0.00085 \\
\hline \multicolumn{11}{|l|}{ Indirect Effect } \\
\hline RSPAN & -- & -- & -- & -- & -- & $-.0021(.0020)$ & $-1.04(112)$ & $>.05$ & & \\
\hline \multirow[t]{2}{*}{ SM } & -- & -- & -- & -- & -- & $.085(.18)$ & $.49(112)$ & $>.05$ & & \\
\hline & & & & & & & Model & $>.05$ & $(2,112)=.59$ & 0.011 \\
\hline & \multicolumn{5}{|c|}{ Mediator } & \multicolumn{5}{|c|}{ Dependent Measure } \\
\hline & \multicolumn{5}{|c|}{ Source Monitoring (SM) } & \multicolumn{5}{|c|}{ False Recognition } \\
\hline Predictor(s) & $b(S E)$ & $t(d f)$ & $p$ & $F(d f)$ & Multiple $r 2$ & $b(S E)$ & $t(d f)$ & $p$ & $F(d f)$ & Multiple $r 2$ \\
\hline \multicolumn{11}{|l|}{ Direct Effects } \\
\hline RSPAN & $0.0020(.0011)$ & $1.85(113)$ & $=0.066$ & $(1,113)=3.44$ & 0.030 & $-0.0019(.0014)$ & $-1.37(113)$ & $>.05$ & $(1,113)=1.86$ & 0.016 \\
\hline SM & -- & -- & -- & -- & -- & $0.20(.12)$ & $1.64(113)$ & $=0.10$ & $(1,113)=2.70$ & 0.023 \\
\hline \multicolumn{11}{|l|}{ Indirect Effect } \\
\hline RSPAN & -- & -- & -- & -- & -- & $-.0024(.0014)$ & $-1.69(112)$ & $=0.094$ & & \\
\hline SM & -- & -- & -- & -- & -- & $.23(.12)$ & $1.92(112)$ & $=0.057$ & & \\
\hline & & & & & & & Model & $=.065$ & $(2,112)=2.80$ & 0.048 \\
\hline
\end{tabular}

Note. Coefficients are unstandardized. Direct effects represent models with a single predictor and the outcome.

Indirect effects represent models with both predictors entered together. 
Table 4. WMC and Source Monitoring Ability Moderation Models for False Recall and False Recognition

Moderation Multiple Regression Summary Table

\begin{tabular}{|c|c|c|c|c|c|c|c|}
\hline \multirow[b]{2}{*}{ Predictors(s) } & \multicolumn{4}{|c|}{ False Recall } & & & Model Comparison \\
\hline & $b$ & $t(d f)$ & $p$ & $F(d f)$ & Multiple $r 2$ & $F(d f)$ & $p$ \\
\hline \multicolumn{8}{|l|}{ Main Effects } \\
\hline RSPAN & $-0.0021(.0020)$ & $-1.04(112)$ & $>.05$ & & & & \\
\hline \multirow[t]{2}{*}{ SM } & $0.085(.18)$ & .49 (112) & $>.05$ & & & & \\
\hline & & Model & $>.05$ & $(2,112)=0.59$ & 0.011 & & \\
\hline \multicolumn{8}{|l|}{$\begin{array}{l}\text { Main Effects }+ \\
\text { Interaction }\end{array}$} \\
\hline RSPAN & $-0.0044(.0088)$ & $-.50(111)$ & $>.05$ & & & & \\
\hline SM & $0.084(.18)$ & $.48(111)$ & $>.05$ & & & & \\
\hline \multirow[t]{3}{*}{ RSPAN*SM } & $0.0034(.012912)$ & $.26(111)$ & $>.05$ & & & & \\
\hline & & Model & $>.05$ & $(3,111)=0.41$ & 0.011 & & \\
\hline & & & & & & $(1,111)=.068$ & $>.05$ \\
\hline
\end{tabular}

\begin{tabular}{|c|c|c|c|c|c|c|c|}
\hline \multirow[b]{2}{*}{ Predictors(s) } & \multicolumn{6}{|c|}{ Dependent Measure } & \\
\hline & $b$ & $t(d f)$ & $p$ & $F$ & Multiple $r 2$ & $F(d f)$ & $p$ \\
\hline \multicolumn{8}{|l|}{ Main Effects } \\
\hline RSPAN & $-0.0024(.0014)$ & $-1.69(112)$ & $=0.094$ & & & & \\
\hline \multirow[t]{2}{*}{ SM } & $0.234(.12)$ & $1.64(112)$ & $=0.057$ & & & & \\
\hline & & Model & $=0.065$ & $(2,112)=2.80$ & 0.048 & & \\
\hline \multicolumn{8}{|l|}{$\begin{array}{l}\text { Main Effects + } \\
\text { Interaction }\end{array}$} \\
\hline RSPAN & $-0.016(.0060)$ & $-2.67(111)$ & $<.01$ & & & & \\
\hline SM & $0.23(.12)$ & $1.91(111)$ & $=0.059$ & & & & \\
\hline \multirow[t]{3}{*}{ RSPAN*SM } & $0.020(.0087)$ & $2.33(111)$ & $<.05$ & & & & \\
\hline & & Model & $<.05$ & $(3,111)=3.75$ & 0.092 & & \\
\hline & & & & & & $(1,111)=5.43$ & $<.05$ \\
\hline
\end{tabular}

Note. Coefficients are unstandardized. Main effects represent models with both predictors entered together.

Main effects plus interaction represent models with predictors and their interaction entered simultaneously.

Model comparison indicates a significance test of the model variance between the main effects and the main effects plus interaction models. 


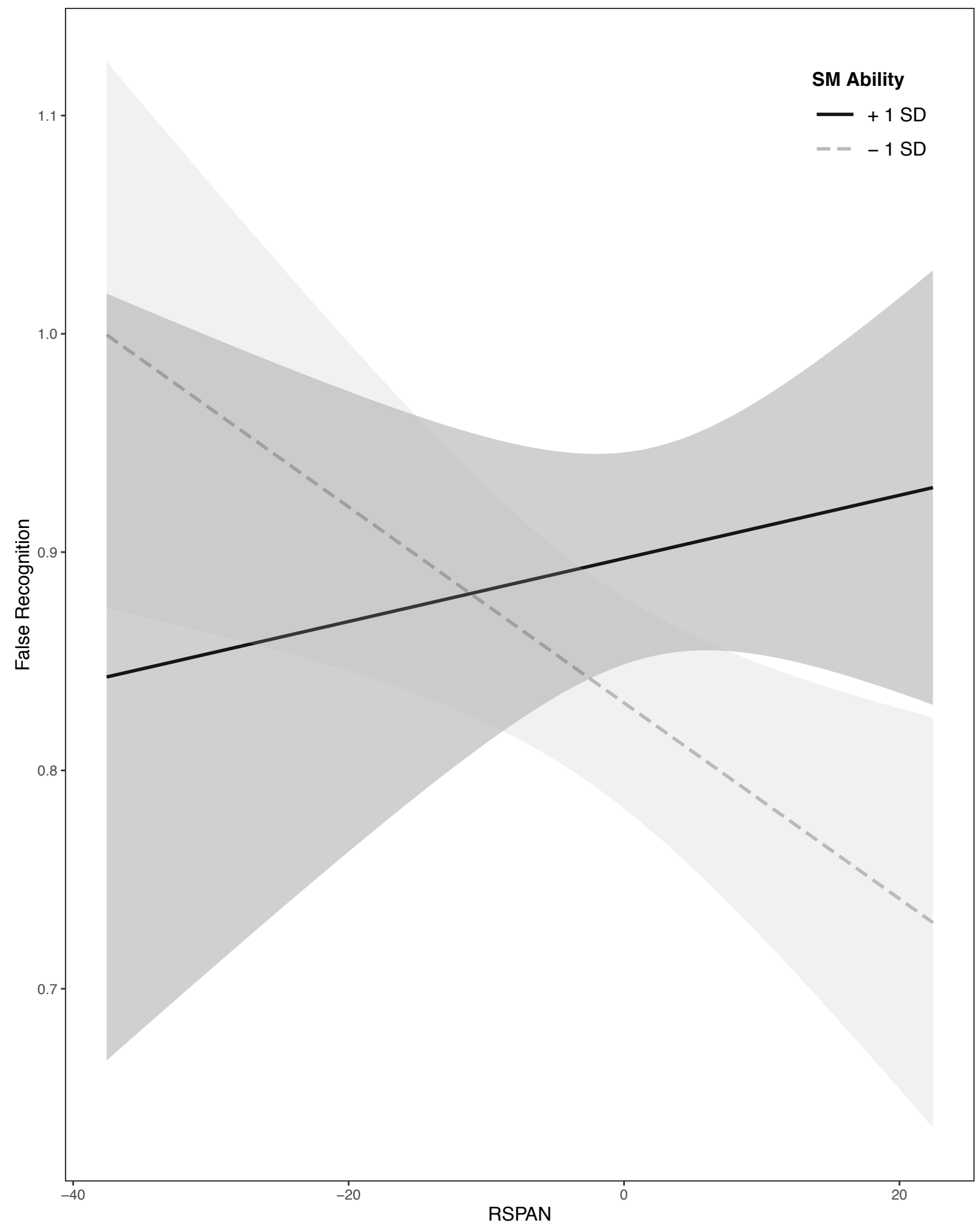

Figure 1. WMC (via RSPAN) and Source Monitoring Ability Moderating False Recognition. 


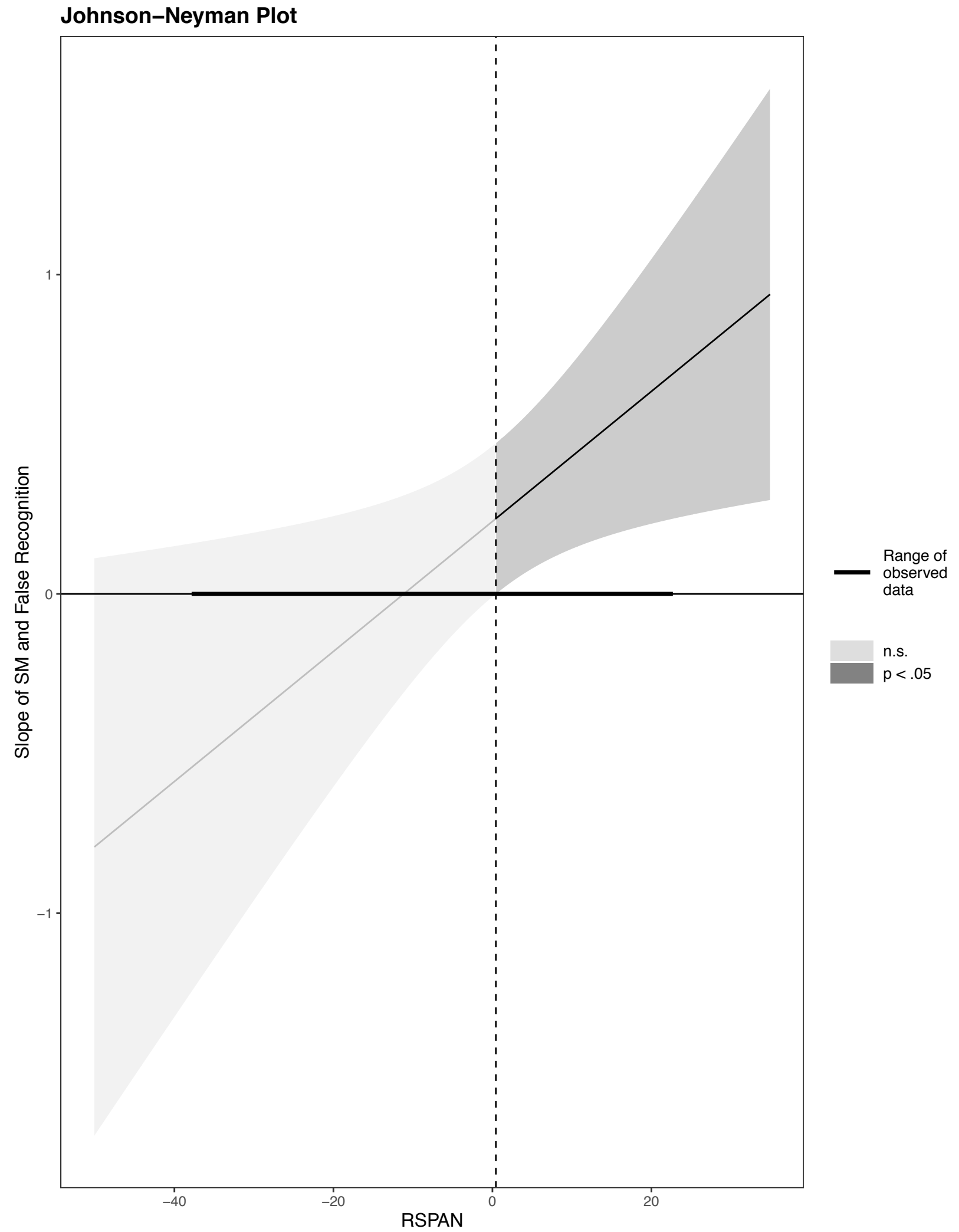

Figure 2. Significance of Slope of SM and False Recognition by WMC (via RSPAN). 